\title{
The structure of linear PF-Engel groups in characteristic zero
}

\author{
B. A. F. Wehrfritz ${ }^{1}$
}

Received: 24 October 2018 / Accepted: 27 February 2019 / Published online: 19 March 2019

(c) The Author(s) 2019

\begin{abstract}
Suppose $\mathrm{G}$ is linear group. If $\mathrm{G}$ has characteristic zero, we prove that $\mathrm{G}$ is (polycyclic-byfinite)-Engel if and only if $\mathrm{G}$ has a normal series $<1>=\mathrm{T}_{0} \leq \mathrm{T}_{1} \leq \cdots \leq \mathrm{T}_{\mathrm{s}}=\mathrm{T} \leq \mathrm{G}$ with $s$ and the index (G:T) finite and each $\mathrm{T}_{\mathrm{i}} / \mathrm{T}_{\mathrm{i}-1}$ either polycyclic-by-finite, or G-hypercentral with $\left[T_{i}, T\right] \leq T_{i-1}$, or G-hypercentral, abelian and Chernikov. This is much more complex than the positive characteristic case where $G$ is (polycyclic-by-finite)-Engel if and only if $G$ is (polycyclic-by-finite)-by-hypercentral.
\end{abstract}

Keywords Engel conditions $\cdot$ Linear group $\cdot$ Matrix group

Mathematics Subject Classification $20 \mathrm{~F} 45 \cdot 20 \mathrm{H} 20$

If $\mathbf{X}$ is a class of groups, say that a group $\mathrm{G}$ is $\mathbf{X}$-Engel if for each $\mathrm{g} \in \mathrm{G}$ there is an $\mathbf{X}$-subgroup $\mathrm{E}$ of $\mathrm{G}$ such that for all $\mathrm{x}$ in $\mathrm{G}$ there is a positive integer $\mathrm{m}$ such that for all $\mathrm{n} \geq \mathrm{m}$ we have $\left[\mathrm{x},{ }_{\mathrm{n}} \mathrm{g}\right] \in \mathrm{E}$. Let $\mathbf{F}$ denote the class of finite groups, $\mathbf{C h}$ the class of Chernikov groups and $\mathbf{P}$ the class of polycyclic groups, so $\mathbf{P F}$ denotes the class of polycyclic-by-finite groups.

A special case of Shumyatsky's main theorem in [4] says that a linear group $G$ is F-Engel if and only if $\mathrm{G}$ is finite-by-hypercentral. By Theorem $\mathrm{Ch}$ of [7] a linear group $\mathrm{G}$ is Ch-Engel if and only if G is Chernikov-by-hypercentral and by Theorem PF of [7] a linear group G of positive characteristic is PF-Engel if and only if G is $\mathbf{P F}$-by-hypercentral (Ch is exactly the class of linear groups satisfying the minimal condition on subgroups and PF is exactly the class of linear groups satisfying the maximal condition on subgroups, so for linear groups $\mathbf{C h}$ and $\mathbf{P F}$ are fully duals of each other).

The situation for linear PF-Engel groups of characteristic zero is much more complex and in particular the obvious analogue of the positive characteristic case is false, see Example 2 below. In this present paper we study in detail the characteristic zero case.

Thus let $\mathrm{G}$ be a PF-Engel subgroup of GL(n, F) for $\mathrm{n}$ some positive integer and $\mathrm{F}$ a field of characteristic 0 . Then $\mathrm{G}$ is soluble-by-finite by Theorem PF of [7]. Let $\mathrm{T}$ be a triangularizable normal subgroup of $G$ of finite index (which always exists, e.g. use [6] 4.2) and denote the unipotent radical of $\mathrm{T}$ by $\mathrm{U}$. Enlarging $\mathrm{F}$ if necessary and replacing $\mathrm{G}$ by a

B. A. F. Wehrfritz

b.a.f.wehrfritz@qmul.ac.uk

1 Queen Mary University of London, London E1 4NS, England, UK 
conjugate, we may assume that $\mathrm{T} \leq \operatorname{Tr}(\mathrm{n}, \mathrm{F})$. Note that $\mathrm{U}$ is nilpotent, $\mathrm{T} / \mathrm{U}$ is abelian and $\mathrm{G} / \mathrm{T}$ is finite.

Theorem With the hypotheses and notation above $G$ has a normal series

$$
<1>=\mathrm{U}_{0} \leq \mathrm{V}_{1} \leq \mathrm{U}_{1} \leq \mathrm{V}_{2} \leq \mathrm{U}_{2} \leq \cdots \leq \mathrm{V}_{\mathrm{t}} \leq \mathrm{U}_{\mathrm{t}}=\mathrm{U} \leq \mathrm{N} \leq \mathrm{T} \leq \mathrm{G},
$$

where $\mathrm{t}=\mathrm{n}(\mathrm{n}-1) / 2$ and for each $\mathrm{i} \geq 1$,

$U_{i} / U_{i-1}$ is central in $U$,

$T / C_{T}\left(U_{i} / U_{i-1}\right)$ is finitely generated,

$V_{i} / U_{i-1}$ is finitely generated abelian,

$U_{i} / V_{i}$ is $G$-hypercentral and

either $U_{i} / U_{i-1}$ is T-central or $U_{i} / V_{i}$ is divisible, abelian and Chernikov.

Further $\mathrm{N}$ is nilpotent of class $\max \{1, \mathrm{n}-1\} \leq \mathrm{n}, \mathrm{T} / \mathrm{N}$ is finitely generated abelian, G/N is polycyclic-by-finite and there exists $M$ a normal subgroup of $G$ such that $U \leq M, M / U$ is (finitely generated abelian)-by-finite and $\mathrm{G} / \mathrm{M}$ is hypercentral.

Corollary Suppose $G$ is linear group of characteristic zero. Then $G$ is PF-Engel if and only if $G$ has a normal series $<1>=\mathrm{T}_{0} \leq \mathrm{T}_{1} \leq \cdots \leq \mathrm{T}_{\mathrm{s}}=\mathrm{T} \leq \mathrm{G}$ with $\mathrm{s}$ and the index (G:T) finite and each $T_{i} / T_{i-1}$

either polycyclic-by-finite,

or G-hypercentral with $\left[T_{i}, T\right] \leq T_{i-1}$,

or G-hypercentral, abelian and Chernikov.

For if $\mathrm{G}$ is PF-Engel take the series $<1>=\mathrm{U}_{0} \leq \mathrm{V}_{1} \leq \cdots \leq \mathrm{U}_{\mathrm{t}} \leq \mathrm{M} \cap \mathrm{T} \leq \mathrm{T} \leq \mathrm{G}$ of the Theorem with $s=2 t+2$. For the converse see Proposition 2 below.

We give examples showing in particular that the group $\mathrm{G}$ in the theorem above need not be PF-by-hypercentral and, unlike the positive characteristic case (see [7]), need not be nilpotent-by-finite.

Below if $\mathrm{G}$ is any group $\left\{\zeta_{\mathrm{i}}(\mathrm{G}): 0 \leq \mathrm{i} \leq \mathrm{s}\right\}$, s some ordinal, denotes the upper central series of $\mathrm{G}$ with $\zeta(\mathrm{G})=\bigcup_{\mathrm{i}} \zeta_{\mathrm{i} \leq \mathrm{s}}(\mathrm{G})$ its hypercentre.

Proposition 1 Let $F$ be a field of characteristic 0, A a non-trivial subgroup of the multiplicative group $F^{*}$ of $F$ and $B$ a non-zero subgroup of the additive group $F^{+}$of $F$ such that $B A=B$. Suppose the split extension $G$ of $B$ by $A$ is $\boldsymbol{P F}$-Engel. Then $G$ is (finitely generated abelian)-by-hypercentral. Further $A$ is finitely generated and $B$ is minimax. In fact $B$ contains a finitely generated subgroup $E$ normal in $G$ such that rankE $=r a n k B$ and $B / E$ is Chernikov and G-hypercentral.

Proof Suppose first that $\mathrm{A}=<\mathrm{a}>$ is cyclic. By hypothesis there is a finitely generated (and abelian note) subgroup $E$ of $B$ such that for all $b$ in $B$ there exists an integer $m(b) \geq 1$ such that for all $n \geq m(b)$ we have $b(a-1)^{n} \in E$. Subject to this we choose our E of least possible rank. Set $\mathrm{E}_{1}=\left\langle\mathrm{b}(\mathrm{a}-1)^{\mathrm{n}}: \mathrm{n} \geq \mathrm{m}(\mathrm{b}), \mathrm{b} \in \mathrm{B}>\leq \mathrm{E}\right.$. Then $\mathrm{E}_{1}$ is also finitely generated, so we may assume that $\mathrm{E}=\mathrm{E}_{1}$. Clearly now $\mathrm{E}(\mathrm{a}-1) \leq \mathrm{E}$.

Since $\mathrm{a} \neq 1$, so $\mathrm{a}-1 \in \mathrm{F}^{*}$ is an (additive) automorphism of $\mathrm{F}^{+}$. Thus $\mathrm{E}(\mathrm{a}-1) \cong \mathrm{E}$ and

$$
\cdots \geq \mathrm{E}(\mathrm{a}-1)^{-\mathrm{i}} \geq \cdots \geq \mathrm{E}(\mathrm{a}-1)^{-1} \geq \mathrm{E} \geq \mathrm{E}(\mathrm{a}-1) \geq \cdots \geq \mathrm{E}(\mathrm{a}-1)^{\mathrm{i}} \geq \cdots
$$


where $i$ runs over the positive integers. Set $E_{2}=\bigcup_{i \geq 0} E(a-1)^{-i}$. If $b \in B$, then for some $\mathrm{n} \geq 1$ we have $\mathrm{b}(\mathrm{a}-1)^{\mathrm{n}} \in \mathrm{E}$, so $\mathrm{b} \in \mathrm{E}(\mathrm{a}-1)^{-\mathrm{n}}$. Therefore $\mathrm{B} \leq \mathrm{E}_{2}$. Further $\mathrm{E}(\mathrm{a}-1) \leq \mathrm{E}$ yields that $\mathrm{Ea} \leq \mathrm{E}$. Also $\mathrm{BA}=\mathrm{B}$, so $\mathrm{E}_{3}=\mathrm{EA}=\bigcup_{\mathrm{i} \geq 0} \mathrm{Ea}^{-\mathrm{i}} \leq \mathrm{B}$. Suppose $\mathrm{b} \in \mathrm{B}$ with ba $\in$ E. There exists $n \geq 1$ with $b(a-1)^{n} \in E$. Then $(-1)^{n} b \in E$. If actually $b \in E_{3}$, then $b a^{m} \in$ $\mathrm{E}$ for some $\mathrm{m} \geq 1$, so $\left(\mathrm{ba}^{\mathrm{m}-1}\right) \mathrm{a} \in \mathrm{E}$ and thus $\mathrm{ba}^{\mathrm{m}-1} \in \mathrm{E}$. A trivial induction yields that $\mathrm{b} \in$ $\mathrm{E}$. This shows that $\mathrm{E}_{3}=\mathrm{E}$ and hence $\mathrm{E}$ is a normal subgroup of $\mathrm{G}$. Clearly $\mathrm{B} / \mathrm{E} \leq \mathrm{E}_{2} / \mathrm{E}$ is A-hypercentral (recall here $\mathrm{A}=\langle\mathrm{a}\rangle$ ) and therefore $\mathrm{G} / \mathrm{E}$ is a hypercentral group.

Since we chose $E$ of least rank, so $E / E(a-1)$ finite. But $E(a-1)^{-i} / E(a-1)^{-i+1}$ is isomorphic to $E / E(a-1)$ for each $i$. Therefore $B / E$ is a periodic $\pi$-group for $\pi$ the finite set of prime divisors of the index $(\mathrm{E}: \mathrm{E}(\mathrm{a}-1))$. Also $\mathrm{E}_{2}$ and $\mathrm{B}$ have finite rank equal to the rank of $\mathrm{E}$ and in particular $\mathrm{B} / \mathrm{E}$ has finite rank. Therefore $\mathrm{B} / \mathrm{E}$ is Chernikov. We have now completed the proof of the case where A is cyclic.

Now assume $A$ is finitely generated, say $A=\left\langle a_{1}, a_{2}, \ldots, a_{t}\right\rangle$, where we choose the generators $a_{i}$ non-zero and such that for each $i, a_{i}^{-1}$ equals some $a_{j}$. By the cyclic case above $B$ is minimax and for each $i$ there is a finitely generated subgroup $E_{i}$ of $B$ such that $E_{i} a_{i}=$ $E_{i}, E_{i}\left(a_{i}-1\right) \leq E_{i} B / E_{i}$ is Chernikov and $<a_{i}>$-hypercentral and rankE $E_{i}$ is minimal with these properties and in fact equals rankB.

Set $\mathrm{E}_{*}=\mathrm{E}_{1}+\mathrm{E}_{2}+\cdots+\mathrm{E}_{\mathrm{t}}$. Since $\mathrm{t}$ is finite, $\mathrm{E}_{*}$ is finitely generated and there exists an integer $\mathrm{m} \geq 1$ such that for all $\mathrm{i}$ and all $\mathrm{n} \geq \mathrm{m}$ we have $\mathrm{E}_{*}\left(\mathrm{a}_{\mathrm{i}}-1\right)^{\mathrm{n}} \leq \mathrm{E}_{\mathrm{i}} \leq \mathrm{E}_{*}$. Set

$$
\mathrm{E}_{* *}=<\mathrm{E}_{*} \mathrm{a}_{1}^{\mathrm{e}(1)} \mathrm{a}_{2}^{\mathrm{e}(2)} \ldots \mathrm{a}_{\mathrm{t}}^{\mathrm{e}(\mathrm{t})}: 0 \leq \mathrm{e}(\mathrm{i})<\mathrm{m}, 1 \leq \mathrm{i} \leq \mathrm{t}>.
$$

Then $\mathrm{E}_{* *}$ is finitely generated. Also that $\mathrm{E}_{*}\left(\mathrm{a}_{\mathrm{i}}-1\right)^{\mathrm{m}} \leq \mathrm{E}_{*}$ implies $\mathrm{E}_{*} \mathrm{a}_{\mathrm{i}}^{\mathrm{m}} \leq<\mathrm{E}_{*} \mathrm{a}_{\mathrm{i}}^{\mathrm{e}}$ : $0 \leq \mathrm{e}$ $<\mathrm{m}>$. Consequently $\mathrm{E}_{* *} \mathrm{a}_{\mathrm{i}} \leq \mathrm{E}_{* *}$ for each $\mathrm{i}$. Further $\mathrm{E}_{* *} \mathrm{a}_{\mathrm{i}}^{-1} \leq \mathrm{E}_{* *}$ since $\mathrm{a}_{\mathrm{i}}^{-1}=\mathrm{a}_{\mathrm{j}}$ for some $\mathrm{j}$. Therefore $\mathrm{E}_{* *}$ is an A submodule of $\mathrm{B}$.

Now $\mathrm{X}=\mathrm{G} / \mathrm{E}_{* *}$ is the split extension of $\mathrm{B} / \mathrm{E}_{* *}$ by $\mathrm{A}$ and $\mathrm{A}$ and $\mathrm{B}$ are abelian. If $\mathrm{b} \in \mathrm{B}$, then $b\left(a_{i}-1\right)^{s} \in E_{i}<E_{*} \leq E_{* *}$ for some $s \geq 1$. Thus the $a_{i}$ are left Engel elements of $X$, as clearly so are the elements of $\mathrm{B} / \mathrm{E}_{* *}$. Trivially $\mathrm{X}$ is abelian-by-polycyclic. Therefore $\mathrm{X}$ is hypercentral by Gruenberg's Theorems 1.2 and 1.4 in [2]. That is, $\mathrm{G}$ is (finitely generated abelian)-by-hypercentral. Finally $\mathrm{B} / \mathrm{E}_{1}$ is Chernikov, so $\mathrm{B} / \mathrm{E}_{* *}$ is too and $\mathrm{rank \textrm {E } _ { * * }} \leq \mathrm{rankB}$ $=\operatorname{rankE} E_{1} \leq \operatorname{rankE} E_{* *}$, so rank $E_{* *}=\operatorname{rank} \mathrm{B}$. This completes the proof of the A finitelygenerated case.

Finally it remains to prove that $\mathrm{A}$ is always finitely generated. Let $\mathbf{J}$ be the subring of $\mathrm{F}$ generated by $\mathrm{A}$. Then $\mathrm{J}$ is a domain and $\mathrm{B}$ is a faithful $\mathrm{J}$-module. Also B is torsion-free and, by the $A$ cyclic case, minimax. Hence $B$ has a free abelian subgroup $B_{0}$ of rank $r=$ $\operatorname{rankB}$ with $\mathrm{B} / \mathrm{B}_{0}$ a periodic $\pi$-group for some finite set $\pi$ of primes. Let $t$ denote the product of all the elements of $\pi$. Then the tensor product $\mathbf{Z}\left[\mathrm{t}^{-1]} \mathrm{B}\right.$ of $\mathbf{Z}\left[\mathrm{t}^{-1}\right]$ by B over $\mathbf{Z}$ is equal to $\mathbf{Z}\left[\mathrm{t}^{-1}\right] \mathrm{B}_{0}$ and hence is isomorphic to $\mathbf{Z}\left[\mathrm{t}^{-1}\right]^{(\mathrm{r})}$, the free $\mathbf{Z}\left[\mathrm{t}^{-1}\right]$-module of rank $\mathrm{r}$. Therefore $\mathrm{J}$ embeds into the endomorphism ring of this module and hence into the matrix ring $\mathbf{Z}\left[\mathrm{t}^{-1}\right]^{\mathrm{r} \times \mathrm{r}}$.

There exists a finitely generated subring $\mathrm{R} \geq \mathbf{Z}\left[\mathrm{t}^{-1}\right]$ of the complex numbers, an element $\mathrm{x}$ of $\mathrm{GL}(\mathrm{r}, \mathrm{R})$ and a subgroup $\mathrm{A}_{0}$ of $\mathrm{A}$ of finite index such that $\left(\mathrm{A}_{0}\right)^{\mathrm{x}} \leq \operatorname{Tr}(\mathrm{r}, \mathrm{R})$. (We now regard $\mathrm{J} \supseteq \mathrm{A}$ as a subring of $\mathrm{R}^{\mathrm{r} \times \mathrm{r}}$ via the obvious embeddings $\mathrm{J} \leq \mathbf{Z}\left[\mathrm{t}^{-1}\right]^{\mathrm{r} \times \mathrm{r}} \leq \mathrm{R}^{\mathrm{r} \times \mathrm{r}}$.) If $\mathrm{a} \in$ $\mathrm{A}_{0}$ with $\mathrm{a}^{\mathrm{x}}$ unipotent, then $\left(\mathrm{a}^{\mathrm{x}}-1\right)^{\mathrm{r}}=0$ in $\mathrm{R}^{\mathrm{r} \times \mathrm{r}}$ and hence $(\mathrm{a}-1)^{\mathrm{r}}=0$ in the domain $\mathrm{J}$. Consequently $\mathrm{a}=1$ and the unipotent radical of $\left(\mathrm{A}_{0}\right)^{\mathrm{x}}$ is trivial. It follows that $\mathrm{A}_{0}$ is isomorphic to a subgroup of the full diagonal group $\mathrm{D}(\mathrm{r}, \mathrm{R})$ and the latter is isomorphic to the direct product of $r$ copies of the group $U$ of units of $R$. But $R$ is a finitely generated domain so $U$ is finitely generated (e.g. [3]). Therefore $\mathrm{A}_{0}$ and $\mathrm{A}$ are also finitely generated. This completes the proof of Proposition 1. 
Lemma 1 Let $A \leq T$ be normal subgroups of the $\boldsymbol{P F}$-Engel group $G$ such that $A$ is abelian and Chernikov, $A \leq \zeta(T)$ and $G / T$ is finite. Then there exists an integer $r \geq 1$ such that $A(r)$ $=\left\{a \in A: a^{r}=1\right\}$ is finite with $A / A(r) \leq \zeta_{\omega}(G / A(r))$.

Proof Let $\mathrm{g}_{1}, \mathrm{~g}_{2}, \ldots, \mathrm{g}_{\mathrm{t}}$ be a transversal of $\mathrm{T}$ to $\mathrm{G}$. Clearly $\mathbf{P F}$ subgroups of A are finite. Hence there exists an integer $r \geq 1$ such that for each a in $A$ and each $i$ there exists $n \geq 0$ such that $\left[a,{ }_{n} g_{i}\right] \in A(r)$. Now $A \leq \zeta(T)$ and $A=\bigcup_{j \geq 1} A(j)$, so there is a series

$$
\mathrm{A}(\mathrm{r})=\mathrm{A}_{0}<\mathrm{A}_{1}<\cdots<\mathrm{A}_{\mathrm{i}}<\cdots<\mathrm{A}_{\omega}=\mathrm{A},
$$

of length the first infinite ordinal $\omega$ of normal subgroups of $G$ such that each $A_{i} / A_{i-1}$ is finite and T-central. Now $A_{i} / A_{i-1}$ consists of right Engel elements of the (finite) split extension of $A_{i} / A_{i-1}$ by $G / T$. Therefore $A_{i} / A_{i-1} \leq \zeta\left(G / A_{i-1}\right)$ and the claim of the lemma follows.

Lemma 2 Let $A \leq$ T be normal subgroups of the $\boldsymbol{P F}$-Engel group $G$ such that $[A, T]=\langle 1\rangle$ and $G / T$ is finite. Then there is a finitely generated subgroup $B$ of $A$ such that $B$ is normal in $G$ and $A / B \leq \zeta(G / B)$.

Note that necessarily A is abelian so B is necessarily polycyclic.

Proof Let $\mathrm{g}_{1}, \mathrm{~g}_{2}, \ldots, \mathrm{g}_{\mathrm{t}}$ be a transversal of $\mathrm{T}$ to $\mathrm{G}$. For each $\mathrm{i}$ there exists a finitely generated subgroup $E_{i}$ of $A$ such that for all a in $A$ there exists $m \geq 1$ with $\left[a,{ }_{n} g_{i}\right] \in E_{i}$ for all $n \geq m$. Set

$$
\mathrm{B}=<\mathrm{E}_{\mathrm{i}}^{\mathrm{g}}: \mathrm{g} \in \mathrm{G}, 1 \leq \mathrm{i} \leq \mathrm{t}>=<\mathrm{E}_{\mathrm{i}}^{\mathrm{g}}: \mathrm{g}=\mathrm{g}_{1}, \ldots, \mathrm{g}_{\mathrm{t}}, 1 \leq \mathrm{i} \leq \mathrm{t}>.
$$

Clearly $\mathrm{B}$ is finitely generated and normal in G. Now A/B consists of right Engel elements of the split extension of $A / B$ by $G / T$. Consequently $A / B \leq \zeta(G / B)$, e.g. [2] Theorems 1.2 and 1.4).

Proof of the Theorem There is a central series $<1>=\mathrm{U}_{0} \leq \mathrm{U}_{1} \leq \ldots \leq \mathrm{U}_{\mathrm{t}}=\mathrm{U}$ of $\mathrm{U}$ and normal in $\mathrm{T}$, where $\mathrm{t}=\mathrm{n}(\mathrm{n}-1) / 2$ and such that each $\mathrm{U}_{\mathrm{i}} / \mathrm{U}_{\mathrm{i}-1}$ embeds into $\mathrm{F}^{+}$so that the action of $\mathrm{T}$ on $\mathrm{U}_{\mathrm{i}} / \mathrm{U}_{\mathrm{i}-1}$ is given by a subgroup of $\mathrm{F}^{*}$ and a homomorphism of it onto $\mathrm{T} /$ $\mathrm{C}_{\mathrm{T}}\left(\mathrm{U}_{\mathrm{i}} / \mathrm{U}_{\mathrm{i}-1}\right)$. Thus for each i Proposition 1 is applicable. Therefore we have

a normal series $<1>=\mathrm{U}_{0} \leq \mathrm{V}_{1} \leq \mathrm{U}_{1} \leq \mathrm{V}_{2} \leq \mathrm{U}_{2} \leq \ldots \leq \mathrm{V}_{\mathrm{t}} \leq \mathrm{U}_{\mathrm{t}}=\mathrm{U}$ of $\mathrm{T}$ such that for each $\mathrm{i}$

$\mathrm{U}_{\mathrm{i}} / \mathrm{U}_{\mathrm{i}-1}$ is central in $\mathrm{U}$,

$\mathrm{T} / \mathrm{C}_{\mathrm{T}}\left(\mathrm{U}_{\mathrm{i}} / \mathrm{U}_{\mathrm{i}-1}\right)$ is finitely generated,

$\mathrm{V}_{\mathrm{i}} / \mathrm{U}_{\mathrm{i}-1}$ is finitely generated abelian,

$\mathrm{U}_{\mathrm{i}} / \mathrm{V}_{\mathrm{i}}$ is $\mathrm{T}$-hypercentral and

either $\mathrm{U}_{\mathrm{i}} / \mathrm{U}_{\mathrm{i}-1}$ is $\mathrm{T}$-central or $\mathrm{U}_{\mathrm{i}} / \mathrm{V}_{\mathrm{i}}$ is divisible, abelian and Chernikov.

Now G/T is finite. Replacing each subgroup in the above series by its normal closure in $\mathrm{G}$ produces a new series that preserves the conditions above. Let us keep the same notation for this new series. Thus we now have the series exactly as above but with the added bonus that the terms are all normal in $\mathrm{G}$.

Suppose $\mathrm{U}_{\mathrm{i}} / \mathrm{V}_{\mathrm{i}}$ is divisible, abelian, Chernikov and T-hypercentral. Then by Lemma 1 there exists $\mathrm{V}_{\mathrm{i}} \leq \mathrm{W}_{\mathrm{i}} \leq \mathrm{U}_{\mathrm{i}}$ with $\mathrm{W}_{\mathrm{i}}$ normal in $\mathrm{G}, \mathrm{W}_{\mathrm{i}} / \mathrm{V}_{\mathrm{i}}$ finite and $\mathrm{U}_{\mathrm{i}} / \mathrm{W}_{\mathrm{i}}$ G-hypercentral. Then $\mathrm{W}_{\mathrm{i}} / \mathrm{U}_{\mathrm{i}-1}$ is finitely generated abelian. Replace $\mathrm{V}_{\mathrm{i}}$ by $\mathrm{W}_{\mathrm{i}}$; that is, assume $\mathrm{V}_{\mathrm{i}}=\mathrm{W}_{\mathrm{i}}$. If 
$\mathrm{U}_{\mathrm{i}} / \mathrm{U}_{\mathrm{i}-1}$ is $\mathrm{T}$-central then by Lemma 2 there exists $\mathrm{U}_{\mathrm{i}-1} \leq \mathrm{W}_{\mathrm{i}} \leq \mathrm{U}_{\mathrm{i}}$ with $\mathrm{W}_{\mathrm{i}}$ normal in $\mathrm{G}$, $\mathrm{W}_{\mathrm{i}} / \mathrm{U}_{\mathrm{i}-1}$ finitely generated and $\mathrm{U}_{\mathrm{i}} / \mathrm{W}_{\mathrm{i}} \mathrm{G}$-hypercentral. Again set $\mathrm{V}_{\mathrm{i}}=\mathrm{W}_{\mathrm{i}}$.

Now $T / C_{T}\left(U_{i} / U_{i-1}\right)$ is finitely generated for each i. Set $\left.N=I_{i} C_{T}\left(U_{i} / U_{i-1}\right)\right)$. Then $\mathrm{T} \geq \mathrm{N} \geq \mathrm{U}$, the group $\mathrm{N}$ is nilpotent $\left(\mathrm{U}_{0} \leq \mathrm{U}_{1} \leq \cdots \leq \mathrm{U}_{\mathrm{t}} \leq \mathrm{N}\right.$ is a central series of $\left.\mathrm{N}\right)$ and normal in $\mathrm{G}, \mathrm{T} / \mathrm{N}$ is finitely generated abelian and $\mathrm{G} / \mathrm{N} \in \mathbf{P F}$. Also $\mathrm{U}$ is the unipotent radical of $\mathrm{N}$. Hence $\mathrm{N}$ embeds into $\mathrm{U}^{*} \times \mathrm{D}$, where $\mathrm{U}^{*}$ is a unipotent linear group of degree $\mathrm{n}$ and characteristic zero and $\mathrm{D}$ is abelian (diagonalizable in fact). Therefore $\mathrm{U}^{*}$ $\times D$ is nilpotent of class $\max \{1, \mathrm{n}-1\}$ and consequently so is $\mathrm{N}$.

Finally $\mathrm{G} / \mathrm{U}$ is an abelian-by-finite PF-Engel group, so by Lemma 2 (with $\mathrm{A}=\mathrm{T}$ ) there is a normal subgroup $\mathrm{M}_{\mathrm{o}}$ of $\mathrm{G}$ with $\mathrm{U} \leq \mathrm{M}_{\mathrm{o}} \leq \mathrm{T}, \mathrm{M}_{\mathrm{o}} \mathrm{U}$ finitely generated abelian and $T / M_{0} \leq \zeta\left(G / M_{0}\right)$. Then there exists $M \geq M_{0}$ normal in $G$ with $M / M_{o}$ finite and $G / M$ hypercentral, see [1].

Suppose $\mathrm{E} \leq \mathrm{H}$ are subgroups of a group $\mathrm{G}$ and $\mathrm{g} \in \mathrm{G}$. If for each $\mathrm{h}$ in $\mathrm{H}$ there exists a positive integer $\mathrm{m}(\mathrm{h})$ such that $\left[\mathrm{h},{ }_{\mathrm{n}} \mathrm{g}\right] \in \mathrm{E}$ for all $\mathrm{n} \geq \mathrm{m}(\mathrm{h})$, for brevity say $\mathrm{E}$ is a g-sink in $\mathrm{H}$. (This is a slight modification of the terminology used in [4].) If this is so, note that $E_{o}=<\left[h,{ }_{n} g\right]: n \geq m(h), h \in H>\leq E$ is also a g-sink in $H$ and it is easy to see that $\left[\mathrm{E}_{\mathrm{o}}, \mathrm{g}\right] \leq \mathrm{E}_{\mathrm{o}} \leq[\mathrm{H}, \mathrm{g}]$. (If $\mathrm{a}, \mathrm{b} \in \mathrm{E}_{\mathrm{o}}$ with $[\mathrm{a}, \mathrm{g}]$ and $[\mathrm{b}, \mathrm{g}]$ in $\mathrm{E}_{\mathrm{o}}$, then $[\mathrm{ab}, \mathrm{g}]=[\mathrm{a}, \mathrm{g}]^{\mathrm{b}}[\mathrm{b}, \mathrm{g}]$ $\in \mathrm{E}_{\mathrm{o}}$ and $\left[\mathrm{a}^{-1}, \mathrm{~g}\right]=\mathrm{a}[\mathrm{a}, \mathrm{g}]^{-1} \mathrm{a}^{-1} \in \mathrm{E}_{\mathrm{o}}$.)

Proposition 2 Let $<1>=T_{0} \leq T_{1} \leq \cdots \leq T_{s}=T \leq G$ be a normal series of the group $G$ with $s$ and $(G: T)$ finite and each $T_{i} / T_{i-1}$

either polycyclic-by-finite,

or G-hypercentral with $\left[T_{i}, T\right] \leq T_{i-1}$,

or G-hypercentral, abelian and Chernikov.

Then G is PF-Engel.

Proof We induct on $\mathrm{s}$; if $\mathrm{s}=0$ the claim is trivial. Suppose $\mathrm{s} \geq 1$, let $\mathrm{g} \in \mathrm{G}$ and assume there exists $T_{1} \leq X \leq G$ with $X / T_{1}$ in $\mathbf{P F}$ and a $g$-sink in $G / T_{1}$. We choose $X$, as we may, with $[X, g] \leq X$, so also $X^{g} \leq X$. If $T_{1}$ is $\mathbf{P F}$, then $X$ is $\mathbf{P F}$ and also a $g$-sink in G. From now on assume that $T_{1}$ is abelian.

There is a finitely generated subgroup $K$ of $X$ with $T_{1} K=X$. If $K=<k_{1}, k_{2}, \ldots, k_{r}>$, then each $\left[k_{i}, g\right] \in u_{i} K$ for some $u_{i}$ in $T_{1}$. Set $R=\left\langle u_{1}, u_{2}, \ldots, u_{r}>^{G}\right.$, a normal subgroup of $\mathrm{G}$. If $\mathrm{x}, \mathrm{y} \in \mathrm{K}$ with $[\mathrm{x}, \mathrm{g}]$ and $[\mathrm{y}, \mathrm{g}]$ both in $\mathrm{RK}$, then

$[x y g]=[x, g]^{y}[y, g] \in(R K)^{y}(R K)=R K$ and $\left[x^{-1}, g\right]=x[x, g]^{-1} x^{-1} \in R K$. Therefore $[\mathrm{K}, \mathrm{g}] \leq \mathrm{RK}$ and hence $[\mathrm{RK}, \mathrm{g}] \leq \mathrm{RK}$.

Now $K$ is finitely generated, $K /\left(K \cap T_{1}\right) \in \mathbf{P F}$ and $K \cap T_{1}$ is abelian. By a theorem of P. Hall (e.g. 3.10 of [6]) $\mathrm{K} \cap \mathrm{T}_{1}$ is finitely K-generated. If $\left[\mathrm{T}_{1}, \mathrm{~T}\right]=<1>$, since (G: $\mathrm{T}$ ) is finite, so $\mathrm{K} \cap \mathrm{T}_{1}$ and $\mathrm{R}$ are finitely generated (as groups note, not just as normal subgroups). Therefore $R K \in \mathbf{P F}$. If $T_{1}$ is Chernikov (and abelian), then $T_{1}$ has an ascending series of normal subgroups of $\mathrm{G}$ of length at most the first infinite ordinal $\omega$ and with all its factors finite. Hence here $\mathrm{K} \cap \mathrm{T}_{1}$ and $\mathrm{R}$ are finite. Again we obtain that $\mathrm{RK} \in \mathbf{P F}$.

Now assume $T_{1}$ is G-hypercentral. Thus let $<1>=\mathrm{Z}_{0} / \mathrm{R} \leq \mathrm{Z}_{1} / \mathrm{R} \leq \cdots \leq \mathrm{Z}_{\tau} / \mathrm{R}=\mathrm{T}_{1} / \mathrm{R}$ be an ascending central series of $G$ in $T_{1} / R$, so all the $Z_{\sigma}$ are normal in $G$. If $k \in K$, then $[\mathrm{k}, \mathrm{g}] \in \mathrm{RK}$, see above. Let $\mathrm{u} \in \mathrm{T}_{1} \backslash \mathrm{R}$ with $\sigma$ the least ordinal with $\mathrm{u} \in \mathrm{Z}_{\sigma}$. Then $\sigma-1$ exists and $[\mathrm{uk}, \mathrm{g}]=[\mathrm{u}, \mathrm{g}]^{\mathrm{k}}[\mathrm{k}, \mathrm{g}] \in \mathrm{Z}_{\sigma-1} \mathrm{RK}=\mathrm{Z}_{\sigma-1} \mathrm{~K}$. Consequently there exists a positive integer $n$ with $\left[\mathrm{uk},{ }_{\mathrm{n}} \mathrm{g}\right] \in \mathrm{RK}$. We have now shown that if $\mathrm{T}_{1} \in \mathbf{P F}$, then $\mathrm{X}$ is $\mathbf{P F}$ and a 
g-sink in $\mathrm{G}$ and if $\mathrm{T}_{1} \notin \mathbf{P F}$, the RK is $\mathbf{P F}$ and a g-sink in $\mathrm{X}$ and hence in $\mathrm{G}$. Therefore $\mathrm{G}$ is PF-Engel.

Example 1 Let a be the companion matrix of the polynomial $\mathrm{X}^{2}-5 \mathrm{X}+1$ in $\mathrm{GL}(2, \mathbf{Z})$. The split extension $\mathrm{G}_{0}$ of $\mathbf{Q}^{(2)}$ by $<\mathrm{a}>$ embeds into $\mathrm{GL}(3, \mathbf{Q})$ and also into $\operatorname{Tr}(2, \mathbf{C})$. Set $\mathrm{E}=$ $\mathbf{Z}^{(2)} \leq \mathbf{Q}^{(2)}, \mathrm{B}=\Sigma_{\mathrm{i} \in \mathbf{Z}} \mathrm{E}(\mathrm{a}-1)^{-\mathrm{i}}$ and $\mathrm{G}=<\mathrm{a}, \mathrm{B}>\leq \mathrm{G}_{0}$. (Note that $\operatorname{det}(\mathrm{a}-1)=-3$, so a-1 is invertible.) Then $\mathrm{G}$ is linear of characteristic zero and polycyclic-by-hypercentral and hence PF-Engel, but is not PF, is not hypercentral and is not (locally nilpotent)-by-finite.

For $\mathrm{E} \geq \mathrm{E}(\mathrm{a}-1)$; thus $\mathrm{E}(\mathrm{a}-1)^{-\mathrm{i}-1} \geq \mathrm{E}(\mathrm{a}-1)^{-\mathrm{i}}$ for all $\mathrm{i}$ and hence $\mathrm{B}=\bigcup_{\mathrm{i} \geq 0} \mathrm{E}(\mathrm{a}-1)^{-\mathrm{i}}$.. Also $\mathrm{Ea}=\mathrm{E}=\mathrm{Ea}^{-1}$, so $\mathrm{Ba}=\mathrm{B}=\mathrm{Ba}^{-1}$; hence $\mathrm{B}$ is an $<\mathrm{a}>$-submodule of $\mathbf{Q}^{(2)}$ and $\mathrm{G}=\langle\mathrm{a}>\mathrm{B}$. Now $\mathrm{E}$ is normal in $\mathrm{G}$ and, being finitely generated abelian, polycyclic. Clearly G/E is hypercentral. Thus in particular G is PF-Engel.

The eigenvalues of a have infinite order (as does a of course). Thus a, and also $\mathrm{a}^{\mathrm{r}}$ for each $\mathrm{r}$ $\neq 0$, have no non-zero fixed points in $\mathbf{Q}^{(2)}$ and hence none in B. It follows that $\zeta_{1}(\mathrm{G})=<1>$ $=\zeta(\mathrm{G})$. In particular $\mathrm{G}$ is not hypercentral. If $\mathrm{G} \in \mathbf{P F}$, then $\mathrm{B}$ is free abelian, necessarily of rank 2, and then (B:E) is finite. Hence there exists $i$ with $E(a-1)^{-i}=E(a-1)^{-i-1}$, But then $\mathrm{E}=\mathrm{E}(\mathrm{a}-1)^{-1}$ and consequently $\operatorname{det}(\mathrm{a}-1)= \pm 1$. This contradiction shows that $\mathrm{G}$ is not polycyclic-by-finite.

If $\mathrm{G}$ is (locally nilpotent)-by-finite, there exists $r>0$ with $<\mathrm{a}^{\mathrm{r}}>\mathrm{B}$ locally nilpotent. Locally nilpotent linear groups are hypercentral ([5] 8.2). Hence $\mathrm{a}^{\mathrm{r}}$ has a non-trivial fixed point in B. This contradiction shows that $\mathrm{G}$ is not (locally nilpotent)-by-finite. Finally if $\alpha=(5+$ $\sqrt{ } 21) / 2 \in \mathbf{C}$, then $\alpha^{2}=5 \alpha-1$. Set $E=\mathbf{Z} \oplus \mathbf{Z} \alpha \leq \mathbf{C}^{*}$. Then $E \alpha^{-i+1} \leq E \alpha^{-i}$ for all $i$ and if $\mathrm{B}=\bigcup_{\mathrm{i} \geq 0} \mathrm{E}(\alpha-1)^{-\mathrm{i}}$, then $\mathrm{B} \alpha=\mathrm{B}$ and the split extension of $\mathrm{B}$ by $\langle\alpha\rangle$ embeds into $\operatorname{Tr}(2, \mathbf{C})$. It is also isomorphic to the group $\mathrm{G}$ above. Similarly the group $\mathrm{G}_{0}$ also embeds into $\operatorname{Tr}(2, \mathbf{C})$. The construction of Example 1 is complete.

Example 2 Let $\mathrm{a}=\operatorname{diag}(1,-1,1), \mathrm{T}=\operatorname{Tr}_{1}(3, \mathbf{Z}[1 / 2])$ and $\mathrm{G}=<\mathrm{a}>\mathrm{T} \leq \operatorname{Tr}(3, \mathbf{Z}[1 / 2]) \leq$ GL(3, Q). Then G is PF-Engel, linear of characteristic zero, but not PF-by-hypercentral.

For brevity represent $\left(\mathrm{x}_{\mathrm{ij}}\right) \in \mathrm{T}$ by the triple $\left(\mathrm{x}_{21}, \mathrm{x}_{32}, \mathrm{x}_{31}\right)$. Let $\mathrm{x}=(\xi, \eta, \zeta)$ and $\mathrm{y}=(\lambda, \mu, \nu)$ be elements of $\mathrm{T}$. Then an elementary calculation in $\mathrm{G}$ yields that

$$
[\mathrm{y}, \mathrm{ax}]=(-2 \lambda,-2 \mu, 2 \lambda \mu-\mu \xi+\eta \lambda),
$$

and then a simple induction yields for all $\mathrm{d} \geq 0$ that

$$
[\mathrm{y}, \mathrm{d}+1 \text { ax }]=\left( \pm 2^{\mathrm{d}+1} \lambda, \pm 2^{\mathrm{d}+1} \mu, \pm 2^{2 \mathrm{~d}+1} \lambda \mu \pm 2^{\mathrm{d}} \mu \xi \pm 2^{\mathrm{d}} \eta \lambda\right) \text {. }
$$

Thus for all large enough $\mathrm{d}$ we have $\left[\mathrm{y},{ }_{\mathrm{d}+1} \mathrm{ax}\right] \in \mathrm{E}=\operatorname{Tr}_{1}(3, \mathbf{Z})$. Note that $\mathrm{E}$ is polycyclic, being finitely generated nilpotent. If $g \in G$, then $y=[g$, ax] lies in $T$, as does $[g, x]$ and then $\left[\mathrm{g},{ }_{3} \mathrm{x}\right]=1$. Consequently $\mathrm{G}$ is PF-Engel.

Let $\mathrm{N}$ be a normal, finitely generated subgroup of $\mathrm{T}$. We claim that $\mathrm{N} \leq \mathrm{Z}=\zeta_{1}(\mathrm{~T})$. Hence suppose $x=(\xi, \eta, \nu) \in N Z$, so at least one of $\xi$ and $\eta$ is non-zero. Since $T$ is nilpotent $N \cap Z \neq$ $<1>$, so $N \cap Z$ is infinite cyclic and NZ/N is a Prüfer 2-group. If $\xi \neq 0$, then

$$
\left[\left(0,2^{-\mathrm{h}}, 0\right), \mathrm{x}\right]=\left(0,0,2^{-\mathrm{h}} \xi\right),
$$

which does not lie in $\mathrm{N} \cap \mathrm{Z}$ for large enough $\mathrm{h}$. Also if $\eta \neq 0$, then

$$
\left[\mathrm{x},\left(2^{-\mathrm{h}}, 0,0\right)\right]=\left(0,0,2^{-\mathrm{h}} \eta\right),
$$


which again does not lie in $\mathrm{N} \cap \mathrm{Z}$ for large enough $\mathrm{h}$. Thus $\mathrm{xZ}$ and hence no non-trivial element of NZ/Z lies in the centre of the nilpotent group $\mathrm{T} / \mathrm{Z}$. Therefore no such $\mathrm{x}$ exists and $\mathrm{N} \leq \mathrm{Z}$ as claimed.

Finally, suppose $M$ is a normal $\mathbf{P F}$ subgroup of $\mathrm{G}$. If ax $\in \mathrm{M}$, where $\mathrm{x} \in \mathrm{T}$, then [G, ax] $\leq \mathrm{M} \cap \mathrm{T}$ and clearly $[\mathrm{G}, \mathrm{ax}] \mathrm{Z}=[\mathrm{G}, \mathrm{a}] \mathrm{Z}=\mathrm{T}$. But $\mathrm{M} \in \mathbf{P F}$, so $\mathrm{M} \cap \mathrm{T}$ is finitely generated. Hence $\mathrm{M} \cap \mathrm{T} \leq \mathrm{Z}$ by the previous paragraph and so $\mathrm{Z}=\mathrm{T}$, which is false. Therefore no such ax exists, $\mathrm{M} \leq \mathrm{T}$ and $\mathrm{M} \leq \mathrm{Z}$, again by the above. But then $\mathrm{G} / \mathrm{M}$ contains sections that are infinite dihedral and consequently $\mathrm{G} / \mathrm{M}$ cannot be hypercentral. This completes the proof that $\mathrm{G}$ is not PF-by-hypercentral.

Remark The fact that the degree is 3 in Example 2 is significant. If $\mathrm{G}$ is a PF-Engel subgroup of GL(2, F), then G is always PF-by-hypercentral. For if G is irreducible, then G is abelian-by-finite and Lemma 2 and the theorem of [1] apply and if $\mathrm{G}$ is reducible, then $\mathrm{G}$ is triangularizable and the Theorem applies with $\mathrm{t}=1$.

\section{Compliance with ethical standards}

Conflict of interest The author states that there is no conflict of interest.

Open Access This article is distributed under the terms of the Creative Commons Attribution 4.0 International License (http://creativecommons.org/licenses/by/4.0/), which permits unrestricted use, distribution, and reproduction in any medium, provided you give appropriate credit to the original author(s) and the source, provide a link to the Creative Commons license, and indicate if changes were made.

\section{References}

1. de Falco, M., de Giovanni, F., Musella, C., Sysak, Y.P.: On the upper central series of infinite groups. Proc. Am. Math. Soc. 139, 385-389 (2011)

2. Gruenberg, K.W.: The upper central series in soluble groups. Illinois J. Math. 5, 436-466 (1961)

3. Samuel, P.: A propos du théorème des unités. Bull. Sci. Math. 90, 89-96 (1966)

4. Shumyatsky, P.: Almost Engel linear groups. Monatsh. f. Math. 186, 711-719 (2018)

5. Wehrfritz, B.A.F.: Infinite Linear Groups. Springer, Berlin (1973)

6. Wehrfritz, B.A.F.: Group and Ring Theoretic Properties of Polycyclic Groups. Springer, London (2009)

7. Wehrfritz, B.A.F.: Weak Engel conditions on linear groups, Advances in Group Theory and Applications, (2018) (to appear)

Publisher's Note Springer Nature remains neutral with regard to jurisdictional claims in published maps and institutional affiliations. 